\title{
A Chinese Restaurant Game for Distributed Cooperative Caching in Small Cell Networks
}

\author{
Junliang Chen ${ }^{1}$,Gang Wang ${ }^{1}$, Fuxiang Wang ${ }^{1 *}$ \\ ${ }^{1}$ School of Electrical and Information Engineering, Beihang University \\ Beijng, BJ 100191 - P. R. China \\ [e-mail: chenjunliang@buaa.edu.cn] \\ *Corresponding author: Fuxiang Wang
}

Received May 20, 2018; revised August 10, 2018; accepted August 24, 2018;

published January 31, 2019

\begin{abstract}
Wireless content caching in small cell networks has recently been considered as a promising way to alleviate the congestion of the backhaul in emerging heterogenous cellular network. However, how to select files which are cached in SBSs and how to make SBSs work together is an important issue for cooperative cache research for the propose of reducing file download time. In this paper, a Cooperative-Greedy strategy (CGS) among cache-enabled small base stations (SBSs) in small cell network is proposed, in order to minimize the download time of files. This problem is formulated as a Chinese restaurant game.Using this game model, we can configure file caching schemes based on file popularity and the spectrum resources allocated to several adjacent SBSs. Both the existence and uniquencess of a Nash equilibrium are proved. In the theoretical analysis section, SBSs cooperate with each other in order to cache popular files as many as possible near UEs. Simulation results show that the CGS scheme outperforms other schemes in terms of the file-download time.
\end{abstract}

Keywords: Small base station, cooperative caching, Chinese restaurant game

This work is supported by the National Natural Science Foundation of China (No.61271194, No.91438207), and the Foundation for Innovative Research Groups of the National Natural Science Foundation of China (No.61521091). 


\section{Introduction}

According to the Cisco's prediction, total mobile data traffic is expected to reach 46.7 exabytes per month by 2021 [1]. In order to cope with the increase of data volume in wireless communication, content caching at edge nodes, such as small base stations (SBSs) and user equipments (UEs), is an effective solution, especially when they cooperate with each other. Cooperative caching has been becoming a research hotspot, because it maximize the amount of data which is preserved in cache[2-4]. A cooperative caching system consists of UEs, caches, and servers. These caches interacts with each other to provide caching services for the UEs so as to reduce bandwidth usage, server workload, and query latency for user devices. Cooperative cache which is viewed as a key technology of next generation networks will be applied in 5G, Internet of Things (IoT), etc. However, how to choose a SBS for every file to save for the purpose that minimize its access times becomes a big challenge.

To solve these problems, there are some works in this field. Specially, Game theory can be used to build collaborative caching strategies. In [5], a Stackelberg game is formulated to deal with the storage resources occupied by each SBS. Server, which predicts the reaction of SBSs and charge the price for caching, acts as the leader. Meanwhile, SBSs act as followers. Finally, a close-form expressions of storage amount and price for caching are derived at the equilibrium of the game. Vo et al. [6] considered cooperation between service providers (SPs) in caching in light of coalitional game. These SPs tend to form coalitions to reduce their operational cost which is mainly made up of bandwidth-expense. Aiming to maximize the social welfare, a cooperative mechanism based on Vickrey-Clarke-Groves (VCG) auctions is proposed in [7], in which cache servers collaborate with each other by means of trading their cache resources. Oualhaj et al. use coalitions games to make cache nodes cooperate as a coalition in order to deliver contents to destination as quickly as possible in [8]. In [9] an overview of converged mobile networks is provided. This method integrates several network resources into a single mobile network. The article [10] proposes a hierarchical cloud computing architecture to enhance performance by adding a mobile dynamic cloud formed by powerful mobile devices to a traditional general static cloud. In [11], H.J. Zhang et al. investigate the joint uplink sub-channel and power allocation problem in cognitive small cells using cooperative Nash bargaining game theory. It can be seen that an architecture of non-orthogonal multiple access (NOMA) based fog radio access networks is proposed, which can meet the heterogeneous requirements in 5G systems in [12].

So, why game theory can be applied in the field of cooperative cache problem? It is because the existence of network externality, especially externality is negative. That is to say, the behavior of a participant in a game may harm the interests of other game players while maximizing their own profits. This kind of problem exist in cooperative cache works among SBSs. Files with different popularity need to find a SBS to store in which they can 
enjoy more spectrum resource, and the spectrum used by each SBS for transmission is limited. Meanwhile, if a SBS caches too many files with high popularity, the SBS will be congested with high traffic, and this situation is undesirable. The competition for spectrum resources among files shows the negative network externality. So we regard the files as participants in the game, and they all want to shorten download time when they are accessed by users. So for this reason, files prefer to be cached in a SBS which has more spectrum resource and cached a few files which has high popularity. Therefore, the problem of cooperative caching is constructed as a game problem with negative network externality. Chinese restaurant game is a suitable method at this time.

The Chinese restaurant game is proposed to solute the choice of customers in Chinese restaurants when they are choosing a table to sit beside [13]. The Chinese restaurant game is based on the Chinese restaurant process (CRP). In Chinese restaurant process[14], there exists infinite number of tables which has infinite number of seats. Guests enter the restaurant in turn, and he can choose to share a table with other guests or sit alone on a table. The probability that he chooses to sit with others and sit along is controlled by one random parameter. So, this process is used to model unknown distributions with negative externality [13]. In the process of Chinese restaurants, the customers' choice are non-strategic. It means that they follow rules without rational concerns on their own utilities. So, [15] introduced strategic behaviors into Chinese restaurant process. On the basis of considering negative network externality, each user analyzes the decision results of other users to make decisions. For the reason of negative externality and introduction of strategic behaviors in CRP, the number of seats and tables in restaurants ought to be finite in order to reflect the competition among decision makers. They propose a new game, called Chinese Restaurant Game. In this game model, customers consider how many people have sat besides every table. For the propose of improving dining experience, they tend to sit at tables where they can get more space.

In this paper, we propose a Cooperative-Greedy strategy (CGS) as a novel solution to cooperative caching based on Chinese restaurant game in order to minimize the download time of files. We regard all of SBSs as tables in the Chinese restaurant game, and each file that is accessed corresponds to a customer who goes to a restaurant for a meal. We allocate the continuous bandwidth to each SBSs according to Reference Signal Receiving Power (RSRP), and the different bandwidth of the SBSs are equivalents to the different sizes of the tables in the restaurant. Both the existence and uniquencess of a Nash equilibrium are proved. The CGS encourages SBSs cooperate with each other to cache popular files as many as possible near UEs. Hence, the file-download time can decrease.

This paper is organized as follows. In section 2, a mathematical model is established. Section 3 analyzes the existence and uniqueness of Nash equilibrium solution. Section 4 puts forward the cooperative-greedy strategy, and gives the pseudo code description.

In section 5 the simulation results are given based on the change of the number of UEs' access amount and SBSs' cache capability.The results show that CGS performs better than the other two algorithms. Finally, section 6 concludes this paper. 


\section{System Model}

Suppose in a user-intensive public place, the communication scenario is shown in Fig.1. In the case of our consideration, there are $\boldsymbol{K}$ SBSs which have cache. A total of $\boldsymbol{N}$ different files may be requested by users. Users access files from those cache in order to ease the burden of backhaul. We intend to effectively use the cache of SBSs to store files, but each SBS can only cache $\boldsymbol{M}$ files, where $\boldsymbol{M}<<\boldsymbol{N}$. So in order to place the file in cache appropriately, we considered the following parameters.

\subsection{File popularity}

In the case of our consideration, we consider the location of files, firstly. All files are represented by a set $F=\left\{f_{1}, f_{2}, \cdots f_{N}\right\}$ where the value of $f_{i}\left(\forall f_{i} \in F\right)$ represents the location of the ith file saved in SBSs' cache. The file may be placed in the cache of a SBS or in the server, so this relationship is represented by

$$
f_{i}= \begin{cases}s \in[1, K] & \text { if the file is saved in cache } \\ s=K+1 & \text { if the file is saved in sever }\end{cases}
$$

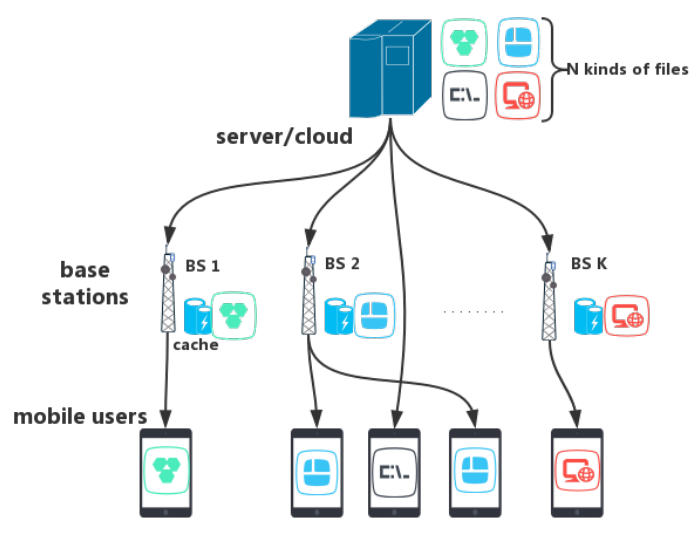

Fig. 1. Communication scene schematic

Then, we need to show the features of files. The files that we consider can be placed in a queue depending on their popularity. The probability denoted by $P=\left\{p_{1}, p_{2}, \cdots p_{N}\right\}$. That the file in the queue is accessed obeys the Zipf distribution [5], i.e.

$$
p_{i}=\frac{\frac{1}{i^{\alpha}}}{\sum_{j=1}^{N} \frac{1}{j^{\alpha}}}
$$


Here, $i$ and $j$ denote the descending position of a file in the popularity table, and $i \neq j$. The greater value of the index $\alpha$, the greater the probability that the file will be reused. When a file is placed in a SBS, popularity indicates the extent to which bandwidth resources are used.

\subsection{The characteristics of communication in reality}

According to the distance between users and SBSs, obstacles and other factors in the real communication scenario, the communication quality of the SBSs in different channels is different. When a user is far away from SBS, the communication quality is poor.

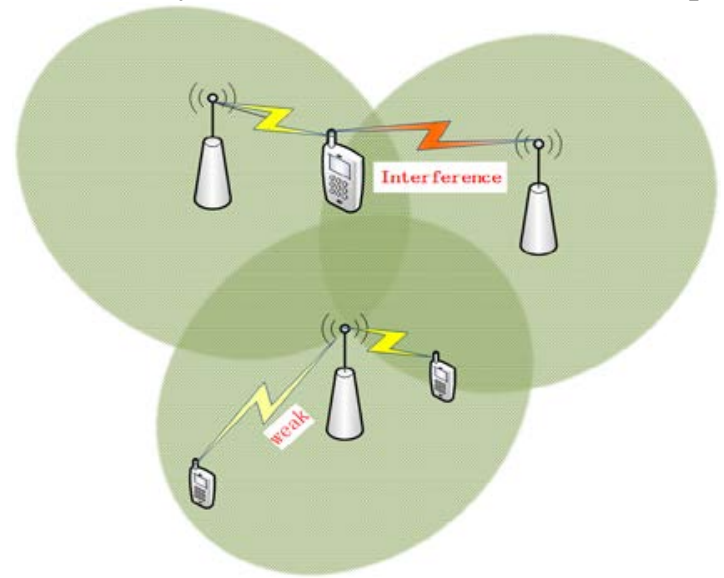

Fig. 2. Communication interference pattern

As shown in Fig. 2, interference from adjacent cell is an important factor affecting communication quality when spectrum is reused. Quality of service (QoE) will be poor when a UE encounters interference from other cells. To eliminate that effect, we use an ideal situation here. We assign each sub-channel in a continuous channel to different SBSs, and the channel resources assigned to each SBS is represented by $B=\left\{b_{1}, b_{2}, \cdots b_{K}\right\}$. The allocation strategy will be detailed in section 4 . Spectrum which is used by a SBS for communication is obtained by Carrier Aggregation(CA).

\subsection{Cooperative-cache strategy}

Based on the Chinese restaurant game model, we propose the Cooperative-cache strategy (CGS). Spectrum allocated to a SBS is counterpart which refers to the size of a table in restaurant. Spectrum collection is denoted by $B$. The situation where a file choose one cache to save is similar to the scenario where a customer in restaurant choose one tale to sit beside. Thus, we define the decision result sets $X=\left\{x_{1}, x_{2}, \cdots x_{K}\right\}$. Denoting the cumulative effect of multi-access, $x_{i}\left(\forall x_{i} \in \mathrm{X}\right)$ which is equal to, in numeral, the sum of file popularity saved in $i$ th SBS. If the value of $x_{i}$ is larger, it is proved that the SBS is under greater access pressure. $B$ and $X$ are important collection in CGS. 
For the purpose of decreasing file download time, we want overall download rate of every file which is cached in SBSs to be as high as possible. It is shown in (3)

$$
R_{\text {sum }}(h, j)=\sum_{j=1}^{N} \text { rate }_{h, j}
$$

For every file $j$, there is a user denoted by $h$ in (3) who wants to download it. If file $j$ is preserved in ith SBS, the download rate of it is shown as following:

$$
\text { rate }_{h, j}=b_{i} \cdot \log _{2}\left(1+\operatorname{SINR}_{i, h}\right)
$$

It is obvious that the rate has positive correlation with $b_{i} \in B$ and Signal to Interference plus Noise Ratio (SINR). The collection $X$ which need to be considered has relation with SINR:

$$
\operatorname{SINR}_{i, h}=\frac{\frac{p_{h}}{d_{i, h}^{\alpha}}}{\sum_{k \in\{\theta\} \backslash h} \frac{p_{k}}{d_{i, k}^{\alpha}}+G N}
$$

In (5), $p_{h}$ is the equipment power of user $h . d_{i, h}$ is the distance between user $h$ and SBS $i$.

$\alpha \in(2,6]$ is the path-loss exponent [16]. $\theta$ is collection of users who are downloading files from SBS $i$. GN is gaussian white noise. The numerator of (5) can be viewed as a constant for a certain user. So, the more users download files from SBS $i$, the worse SINR will become. The reason is existence of congestion. And the extent of congestion in communication can be represented by collection $X . S I N R_{i, h}$ will decrease with the increase of $x_{i} \in X$. So, the correlation among $B, X$ and $R_{\text {sum }}(h, j)$ is that $B$ has positive correlation with $R_{\text {sum }}$ and $X$ has negative correlation with $R_{\text {sum }}$.

Above all, we can construct a function with two variables including $b \in B$ and $x \in X$. We use $U\left(b_{i}, x_{i, j}\right)$ which is utility function of $j$ th file when $j$ th file is going to be cached in $i$ th SBS. $b_{i}$ represents the bandwidth of the SBS where the file is located. $x_{i, j}$ represents the combined popularity of all cached files in ith SBS. The two parameters mentioned above determine the extent of fluency that UEs can enjoy when they download $j$ th files from the SBS.

There are $K$ SBSs to choose by $N$ files which are sorted according to popularity. For all $f_{j}$ in $F$, its cache selection is based on $X_{-j}=\left\{x_{1,-j}, x_{2,-j}, \cdots x_{K,-j}\right\} . X_{-j}$ denotes a collection of storage result of files that are already cached by SBSs when the file $f_{j}$ is not been placed in the base station cache. $f_{j}$ cache selection should obey the following policy:

$$
f_{j}=i, \quad \text { if } i=\arg \max \left[U\left(b_{i}, x_{i,-j}+p_{j}\right)\right]
$$


In other words, based on the cache results of other files, the file $f_{j}$ finds a SBS's cache to save where it can get the maximum utility function value as its own selection result in various available options. The utility function is shown as follows:

$$
U\left(b_{i}, x_{i, j}\right)=\log _{10}\left(\frac{b_{i}}{\sum_{j=1}^{\varepsilon} x_{i, j}}\right)
$$

where $b_{i}$ denotes the sum of sub-channels which are allocated to ith SBS. $\log _{10}(\cdot)$ which is used in (7) is monotone function and it can reduce the absolute value of data which is quiet big for easy calculation.

\section{Theoretical Analysis}

The Nash equilibrium refers to a result combination of various participating decision makers under a non-cooperative game model. Under this model, the people involved in the decision-making cannot improve their own profits without losing at least one other participant's interest. That is the Pareto optimality in Nash Bargaining. In CGS, it is shown that no single file can achieve better utility function value by changing only own decisions. The existence and uniqueness of equilibrium are demonstrated in this chapter.

Theorem 1: For SBSs' bandwidth $B=\left\{b_{1}, b_{2}, \cdots b_{K}\right\}$ file popularity $P=\left\{p_{1}, p_{2}, \cdots p_{N}\right\}$. Any Nash equilibrium result set $X^{*}$ satisfies the following condition:

\section{Proof:}

$$
\begin{aligned}
& U\left(b_{f_{j}}, x_{f_{j}}^{*}\right)>U\left(b_{f_{j}}^{\prime}, x_{f_{j}}^{\prime^{*}}+p_{f_{j}}\right) \\
& \text { s.t. } \forall b_{f_{j}}, b_{f_{j}}^{\prime} \in B ; \forall x_{f_{j}}^{*}, x_{f_{j}}^{\prime^{*}} \in X
\end{aligned}
$$

sufficient condition: If the decisions set made by files is $F=\left\{f_{1}, f_{2}, \cdots f_{N}\right\}$, the resulting set of files location is $X^{*}=\left\{x_{1}^{*}, x_{2}^{*}, \cdots x_{K}^{*}\right\}$ which satisfy the condition of (8). When the $i$ th file selects the $j$ th SBS, and there is $f_{i}=j$. The corresponding utility function meet the condition $U\left(b_{j}, x_{j}^{*}\right)=U\left(b_{f_{i}}, x_{f_{i}}^{*}\right)$. Supposing that the ith file is no longer saved by the SBS $j$ and it is preserved by the SBS $k$, the utility function is $U\left(b_{k}, x_{k}^{*}+p_{i}\right)=U\left(b_{f_{i}}^{\prime}, x_{f_{i}}^{\prime^{*}}+p_{i}\right)$. Because of (8), we know that $U\left(b_{j}, x_{j}^{*}\right)>U\left(b_{k}, x_{k}^{*}+p_{i}\right)$. To maximize the utility function of $f_{i}$, file $i$ should be saved in SBS $j$ unchanged. That is to say $F=\left\{f_{1}, f_{2}, \cdots f_{N}\right\}$ is a Nash equilibrium.

Necessary condition: If there is a Nash equilibrium $F^{*}=\left\{f_{1}^{*}, f_{2}^{*}, \cdots f_{N}^{*}\right\}$, the resulting set is $X^{*}=\left\{x_{1}^{*}, x_{1}^{*}, \cdots x_{K}^{*}\right\}$. There is $f_{i}=j$ as the ith file selects the $j$ th SBS. The utility 
function can be represented by $U\left(b_{j}, x_{j}^{*}\right)=U\left(b_{f_{i}}, x_{f_{i}}^{*}\right)$. When the $i$ th file is saved in SBS $k$ instead of SBS $j$ which is the ith file's former choice, the utility function is $U\left(b_{k}, x_{k}^{*}+p_{i}\right)=U\left(b_{f_{i}}^{\prime}, x^{\prime{ }^{*}} f_{i}+p_{i}\right)$. For the reason that $F^{*}=\left\{f_{1}^{*}, f_{2}^{*}, \cdots f_{N}^{*}\right\}$ is a Nash equilibrium, we can apply Pareto optimality under this case. Then we have $U\left(b_{j}, x_{j}^{*}\right)>U\left(b_{k}, x_{k}^{*}+p_{i}\right)$. In other word, (8) is ture.

From this theorem, we can see that the utility function of a file under the condition of Nash equilibrium cannot be improved by shifting storage location. Also, a file breaking a equilibrium, it will aggravate communication congestion of the SBS in which the file is saved now.

Theorem 2: If (8) is appropriate for ith file and $j$ th file, then Equilibrium result is unique.

Proof:

We use a contradiction. Suppose there are two equilibrium result sets: $X^{\prime}=\left\{x_{1}^{\prime}, x_{2}^{\prime}, \cdots x_{K}^{\prime}\right\}$ and $X^{*}=\left\{x_{1}^{*}, x_{2}^{*}, \cdots x_{K}^{*}\right\}$. Then we have the following results.

$$
\begin{gathered}
\exists x_{i}^{\prime}>x_{i}^{*}, x^{\prime}{ }_{j}<x_{j}^{*} \\
\text { s.t. } \sum_{m=1}^{K} x_{m}^{\prime}=\sum_{m=1}^{K} x_{m}^{*}=1
\end{gathered}
$$

Because $U(\cdot)$ is a decreasing function of $x$, so $\exists p_{t} \in P$ makes that:

$$
\begin{gathered}
U\left(b_{i}, x_{i}^{*}\right)>U\left(b_{i}, x_{i}^{*}+p_{t}\right) \geq U\left(b_{i}, x_{i}^{\prime}\right) \\
U\left(b_{j}, x_{j}^{\prime}\right)>U\left(b_{j}, x^{\prime}{ }_{j}+p_{t}\right) \geq U\left(b_{j}, x_{j}^{*}\right)
\end{gathered}
$$

Since $X^{\prime}$ is also the equilibrium result, according to (8), we have

$$
U\left(b_{i}, x_{i}^{\prime}\right)>U\left(b_{j}, x_{j}^{\prime}+p_{t}\right)
$$

Considering(10), (11), and (12), it can be obtained the following result:

$$
U\left(b_{i}, x_{i}^{*}+p_{t}\right) \geq U\left(b_{i}, x_{i}^{\prime}\right) \geq U\left(b_{j}, x^{\prime}{ }_{j}+p_{t}\right) \geq U\left(b_{j}, x_{j}^{*}\right)
$$

However, the left and right sides of (13) are opposite to (8). So this assumption is invalid. That is to say, the equilibrium result is unique.

\section{Cooperative-Greedy Strategy}

\subsection{Spectrum Allocation}

In order to avoid the interference of spectrum reuse among multi-SBSs with near distance. According to the comparison of the communication quality of each SBS in the sub-channel, the sub-channel of the continuous frequency band is allocated to the SBS with good communication condition. The quality of communication is measured by the Reference Signal Receiving Power(RSRP).

RSRP varies according to distance between SBSs and UEs, space obstacle and other factors. If the set of sub-channels is represented as $C=\left\{c_{1}, C_{2}, \cdots c_{n}\right\}$, which is shown in 
Fig.3, the RSRP of SBS $i$ is $R_{i}=\left\{r_{i, 1}, r_{i, 2}, \cdots r_{i, n}\right\}$, and the spectrum resource occupied by $K$ SBSs is $B=\left\{b_{1}, b_{2}, \cdots b_{K}\right\}$. The sub-channel allocation strategy is:

$$
\begin{gathered}
b_{i}=\sum_{j=0}^{n} c_{j} \cdot s_{j} \\
s_{j}= \begin{cases}1 & \text { if } r_{i, j}=\max \left(r_{1, j}, r_{2, j}, \cdots r_{K, j}\right) \\
0 & \text { others }\end{cases}
\end{gathered}
$$

In order to measure the RSRP, two reference signals need to be selected at each time period, and a reference signal is selected as an evaluation by the six sub-carriers. In the spectrum allocation, we consider the communication state of each mobile device on each sub-carrier when it is served by a certain SBS. By averaging, the SBS obtains the sub-carrier when it has highest RSRP at this sub-carrier.

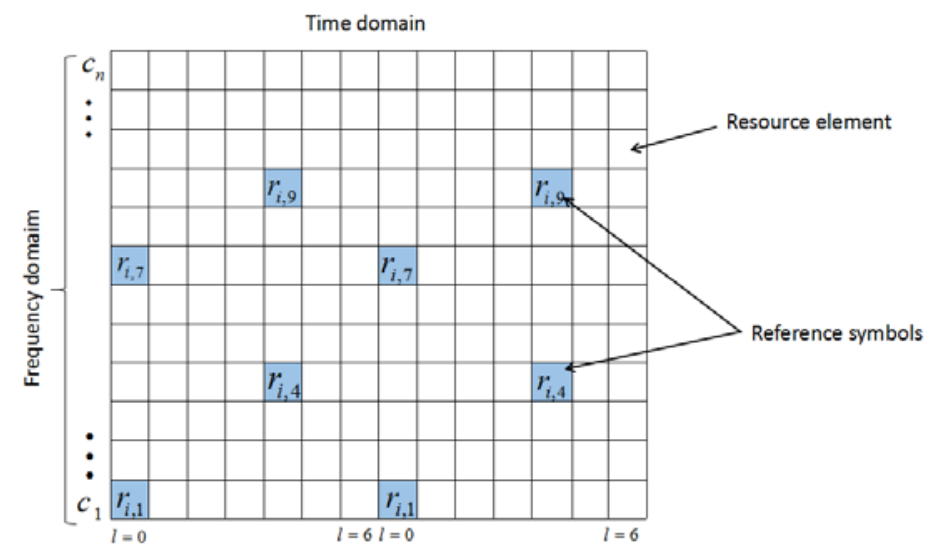

Fig. 3. Reference Signal Receiving Power

\subsection{File Allocation}

The algorithm uses sequential decision making. In CGS, files determine the SBS in which they are placed in sequence. The pseudo code of the program is briefly described in Algorithm 1.

File $i$ check whether the cache of SBS $j$ is full. If the SBS $j$ is not fully occupied, file calculate the value of the utility function that the file will achieve in the case of saving in this SBS. If all SBSs are full, the file is placed on the server. The file compares the value of the utility function when it is stored in a SBS, and selects the SBS that gives it the maximum value.

In this algorithm, every file $i$ needs to search every available SBS in order to find the best placement selection. So the time complexity $\mathrm{T}(\mathrm{n})=\mathrm{o}(\mathrm{NK})$. 


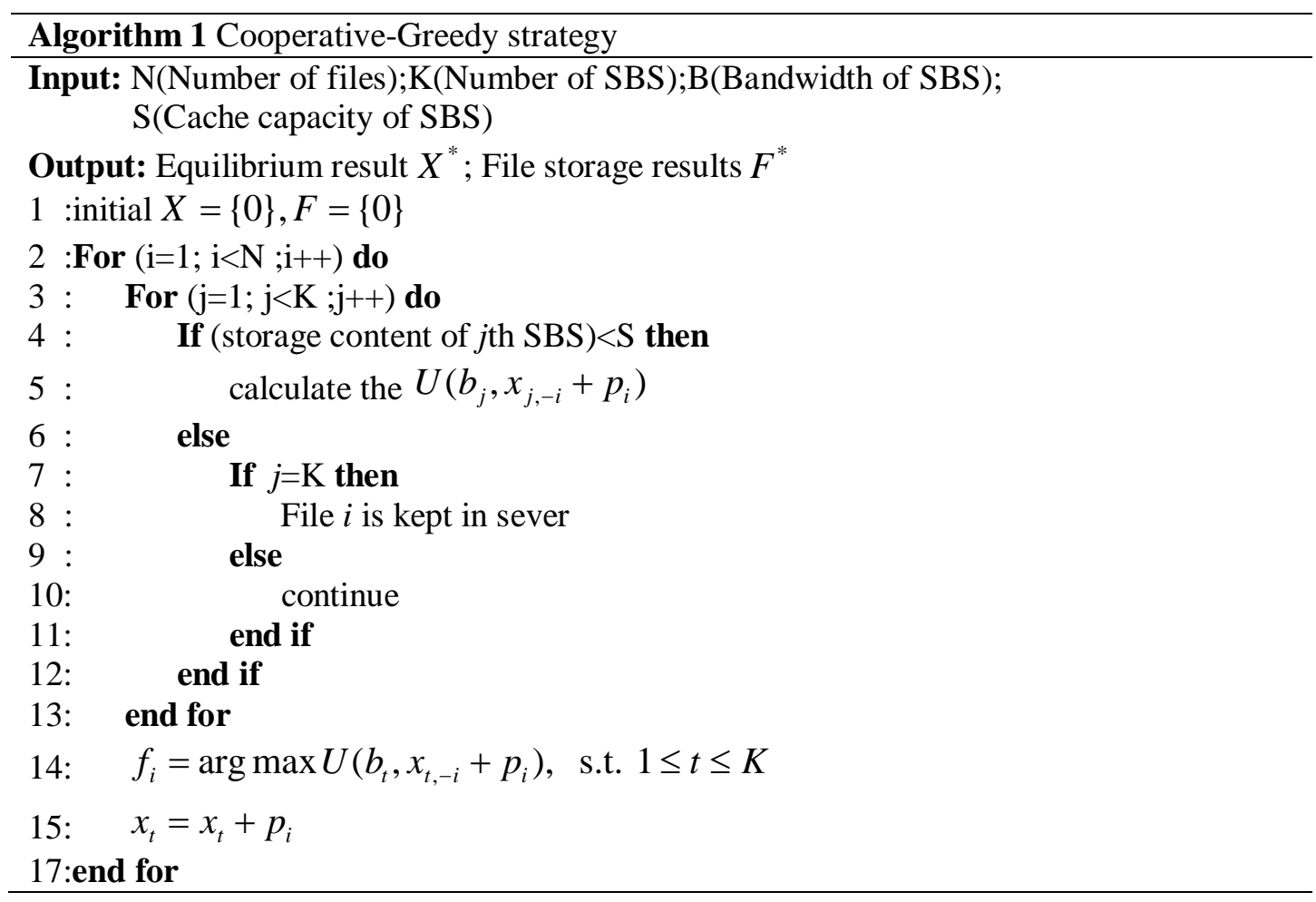

\section{Simulation Results}

We test the performance of the CGS by simulation using two independent variables. The simulation parameter Settings are shown in the Table 1. The download time of files is regarded as the most important dependent variable. The download time is shorter, the better users' experience will be.

Table 1. parameter setting of simulation

\begin{tabular}{|l|l|}
\hline \multicolumn{1}{|c|}{ parameter } & values \\
\hline Carrier bandwidth & $20 \mathrm{MHz}$ \\
\hline Sub-carrier bandwidth & $15 \mathrm{KHz}$ \\
\hline Test area size & $200 \mathrm{~m} * 200 \mathrm{~m}$ \\
\hline Total number of documents & $5000 \mathrm{files}$ \\
\hline Transmit power of users & $20 \mathrm{dBm}$ \\
\hline The rang of RSRP & $-105 \mathrm{dBm} \sim-65 \mathrm{dBm}$ \\
\hline Size of files & $20 \mathrm{M}$ bits \\
\hline
\end{tabular}

We set two other cache strategies as the control groups.

Greedy strategy: keep copies of the most popular files in each SBS. In this case, majority of files are kept in the server, which is a non-cooperative algorithm. 
Round-select strategy: in the form of cooperative cache, files are stored in SBSs in order of popularity.

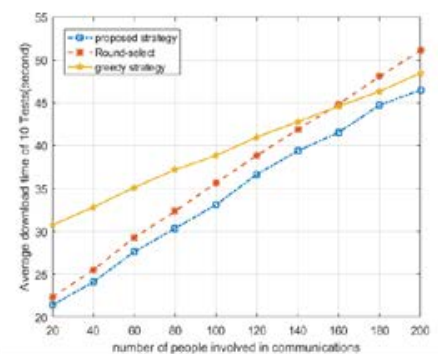

(a)

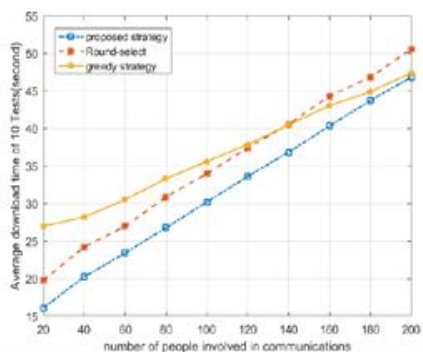

(b)

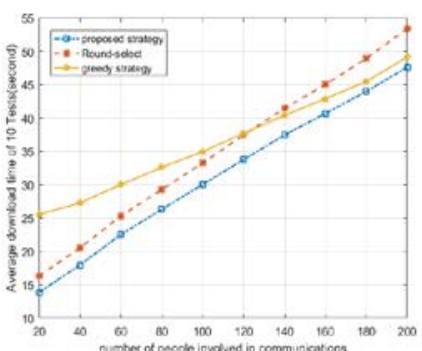

(c)

Fig. 4. change of FDT with the change of NOU

There are three factors which could effect the experience of users who access files from SBSs. The three factors are Number of Users (NOU) who participate communication, Number of SBSs (NOS), and Cache Capability of SBSs (CC) which is represented by number restriction of files saved in SBSs. When variables take different values (e.g. NOU, NOS and CC are 100, 5, 200 separately), the experiment was conducted ten times, and the average was taken in order to prevent the influence of individual unstable values.

In this chapter, we show the influences of these three factors on file-download time (FDT) one by one. In Fig.4, we demonstrate the impact which is imposed by change of NOU on FDT. There are three comparison figures(a, b, and c) in Fig. 4 when CC change from 100, 200 to 300 respectively. It's found that file-download time decreased with the increment of CC, after comparing three insets in Fig. 4. This is because more files are cached in SBSs near users, and the FDT is much shorter when users access files from SBSs than the time cost if people download files from sever.

The variation trend of FDT is same in three insets with the change of NOU. Take a look at inset a. As NOU increase, the congestion get more sever. As a result, FDT goes up. At the same time, it can be seen that the FDT of CGS is shorter than the other two methods, this is because the communication quality and bandwidth of the SBSs are taken into account in CGS when a file is placed. It is also noted that the FDT line of round-select strategy and the greedy strategy will be crossed as the NOU increases. When the NOU is less than 120, the FDT of the round-select strategy is shorter than that of the greedy strategy. As the NOU increases, the performance of the round-select strategy falls behind the greedy strategy. There are two reasons: first, when the NOU is small, the access pressure of each SBS is limited. The round-select strategy cache more files in the SBS compared to greedy method, thus obtaining less FDT. But when the NOU is large, due to file placement choice in the round-select strategy is simple, the SBSs that cache high popularity files will have more people to visit, resulting in serious congestion. Meanwhile, the greedy strategy has better performance than the round-select strategy because multiple SBSs share these access pressures.

It can be seen from figure 4 that CGS has a good adaptability to the increase of the number of users. 


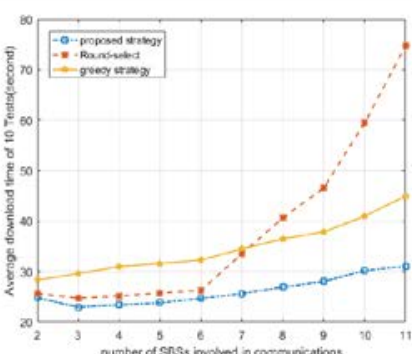

(a)

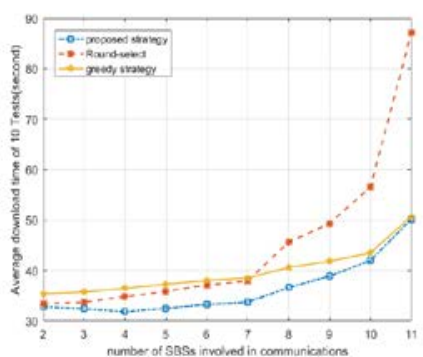

(b)

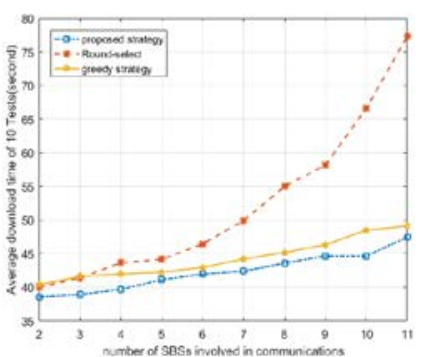

(c)

Fig. 5. change of FDT with the change of NOS

In Fig. 5, we show the change of FDT with the change of NOS. The NOU is 50, 100, 150 receptively in inset a, b and c. By means of comparing these three insets, we can find FDT increases when NOU get larger.

Because the trend of lines in the three insets are similar, we take inset (a) as the representation It can be seen that as the NOS increases, the download time will increase slightly except the round-select strategy, but it remains stable. This is due to the interaction of two factors: The spectrum of fixed width is allocated to more base stations. So the spectrum resource of one SBS is less and the congestion of the communication increases. At the same time, more SBSs are involved in communication so that more files can be cached in the SBSs, which reduces the growth of FDT.

As the NOS increases, the download time will increase rapidly when round-select strategy is applied. This is due to the fact that the round-select strategy does not consider the bandwidth of each SBS when a file choose one SBS to be cached. The SBSs with good communication status and many resources have stored the unfashionable files, and the SBSs with poor communication conditions have stored the files with high popularity. As the NOS increases, the mismatch between resource and access pressure get severe, making the FDT increase. The greedy strategy cached less files in SBSs than the CGS, because there is no collaborative caching pattern. So the performance is not as good as the CGS.

Compared with two other methods, the CGS not only ensure that the spectrum resource allocated to SBSs matches the access pressure but also caches more files in the SBSs.

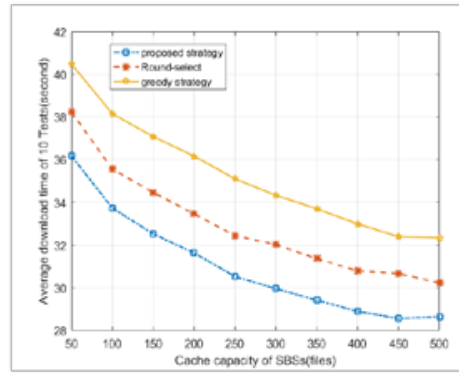

(a)

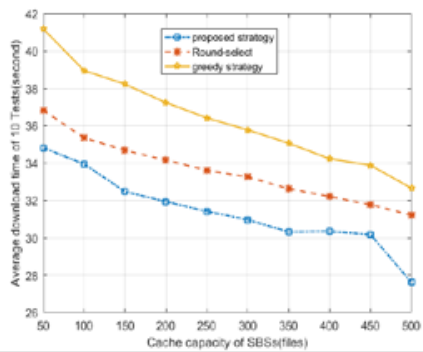

(b)

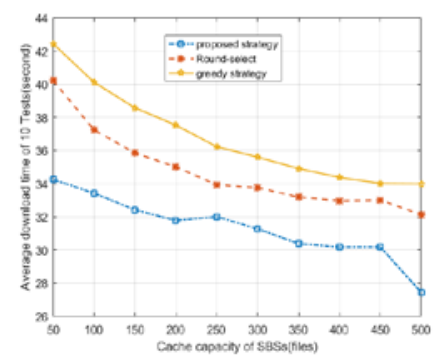

(c)

Fig. 6. change of FDT with the change of CC 
Fig. 6 shows the change of FDT with the increase of SBS cache capacity. The NOS is 2, 3,4 receptively in inset a, b and c. After comparison, as the NOS increased, the download time of the files remained basically unchanged.

It can be seen from the figure that the download time of CGS is the shortest, and the round-select strategy is longer. The reason is that both methods use a collaborative caching approach, which caches more files in SBSs. In greedy strategy, SBSs cache a small amount of files with high popularity. So some users whose FDT is quiet long access files from sever and the performance of greedy strategy is not good.

From Fig. 6, it can be seen that the increase of the SBSs' cache capacity can significantly reduce the file download time, while the CGS can maintain good performance.

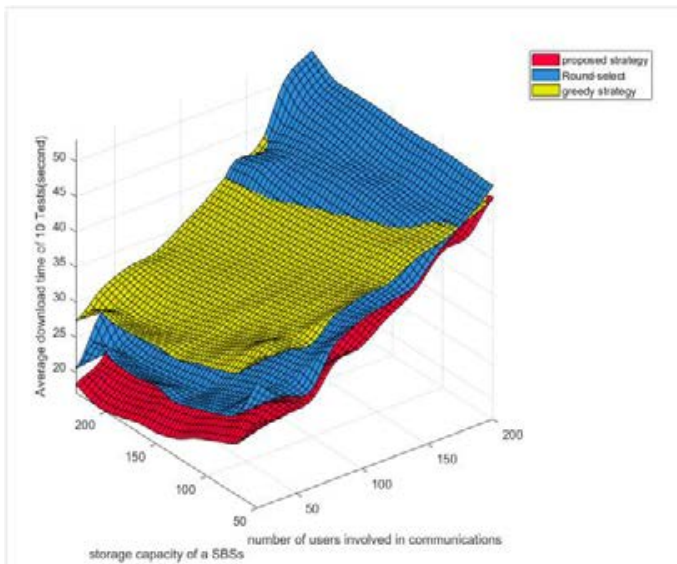

(a)

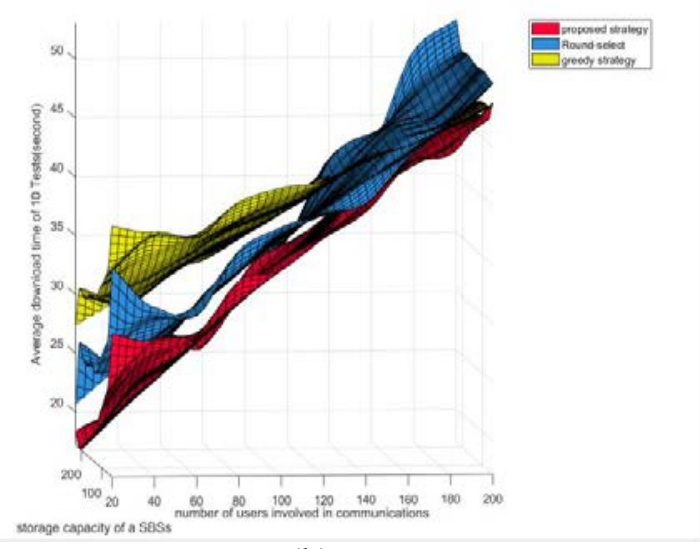

(b)

Fig. 7. change of FDT with two variables

Fig. 7 shows the combined effect of two variables - the number of users and the cache capability on the user's download time. Inset B is the right view. It can be seen that the FDT is positively correlated with the NOS, and is negatively correlated with the CC. When the NOS is large enough, the greedy strategy's performance exceeds the round-select strategy. At the same time, combined with inset b, it can be seen that CGS has always maintained the best performance among three cache strategies, and has a good adaptability to the change of NOS and the changes of the CC.

From the simulation results, it can be seen that CGS adopts the method of cooperative cache, and matches the spectrum resources occupied by the SBSs with the popularity of the files to obtain good performance. It has a good adaptability to the increase of the number of users. Meanwhile, the change of NOS has little effect on CGS performance, and the performance of CGS can be significantly improved when the CC is enhanced. 


\section{Conclusions}

In this paper, we propose the "cooperative-greedy" strategy (CGS) based on the Chinese restaurant game model in field of cooperative cache. The CGS contains two parts: one is to allocate each sub-carrier to SBSs according to the RSRP. The other is that the files which need to be placed in the SBSs are selectively placed according to the bandwidth of SBSs. Based on the mathematical model, the existence and uniqueness of Nash equilibrium solution are analyzed. The simulation results show that the CGS is always able to obtain a shorter download time compared with the other two algorithms. With the increase of the number of users, the number of SBSs and the increase of the SBSs' cache space, the advantages of CGS are more clear, indicating that this caching algorithm has better performance.

\section{References}

[1] Visual Networking Index. Cisco visual networking index: Global mobile data traffic forecast update, 2016-2021 white paper, 2017. Article (CrossRef Link)

[2] Huang, Xueqing, and N. Ansari, "Content Caching and Distribution in Smart Grid Enabled Wireless Networks," IEEE Internet of Things Journal, 4.2, 513-520, 2016. Article (CrossRef Link)

[3] Li, Hongjia, Z. Wang, and D. Hu. "Joint wireless and backhaul load balancing in cooperative caches enabled small-cell networks," in Proc. of IEEE, International Symposium on Personal, Indoor, and Mobile Radio Communications IEEE, 1889-1894, 2015. Article (CrossRef Link)

[4] Li, Hongjia, et al., "Cooperative RAN Caching based on Local Altruistic Game for Single and Joint Transmissions," in IEEE Communications Letters, PP.99:1-1, 2016.

Article (CrossRef Link)

[5] Xu, Xiaodong, J. Liu, and X. Tao, "Mobile Edge Computing Enhanced Adaptive Bitrate Video Delivery With Joint Cache and Radio Resource Allocation," IEEE Access, 5.99:16406-16415, 2017. Article (CrossRef Link)

[6] Vo, Phuong Luu, et al., "A coalitional game approach for fractional cooperative caching in content-oriented networks," Computer Networks the International Journal of Computer \& Telecommunications Networking, 77.C:144-152, 2015. Article (CrossRef Link)

[7] Dai, Jie, et al., "Collaborative Caching in Wireless Video Streaming Through Resource Auctions," IEEE Journal on Selected Areas in Communications 30.2:458-466, 2012.

Article (CrossRef Link)

[8] Oualhaj, Omar Ait, et al., "A coalitional-game-based incentive mechanism for content caching in heterogeneous Delay Tolerant Networks," Wireless Communications and Mobile Computing Conference IEEE, 987-992, 2015. Article (CrossRef Link)

[9] Jo, M., Maksymyuk, T., Batista, R. L., \& Maciel, T. F., “A survey of converging solutions for heterogeneous mobile networks,” IEEE Wireless Communications, 21(6), 54-62, 2014. Article (CrossRef Link)

[10] Jo, M., Maksymyuk, T., Strykhalyuk, B., \& Cho, C. H., "Device-to-device-based heterogeneous radio access network architecture for mobile cloud computing," IEEE Wireless Communications, 22(3), 50-58, 2015. Article (CrossRef Link)

[11] Zhang, H., Jiang, C., Beaulieu, N. C., Chu, X., Wang, X., \& Quek, T. Q. S., "Resource allocation for cognitive small cell networks: a cooperative bargaining game theoretic 
approach,” IEEE Transactions on Wireless Communications, 14(6), 3481-3493, 2015. Article (CrossRef Link)

[12] Zhang, H., Qiu, Y., Long, K., Karagiannidis, G. K., Wang, X., \& Nallanathan, A., "Resource allocation in noma-based fog radio access networks," IEEE Wireless Communications, 25(3), 2018. Article (CrossRef Link)

[13] Wang, Chih Yu, Y. Chen, and K. J. R. Liu, "Chinese Restaurant Game." IEEE Signal Processing Letters, 19.12:898-901, 2014. Article (CrossRef Link)

[14] Pitman, Jim, "Exchangeable and partially exchangeable random partitions," Probability Theory \& Related Fields, 102.2:145-158, 1995. Article (CrossRef Link)

[15] Wang, Chih Yu, Y. Chen, and K. J. R. Liu, "Sequential Chinese Restaurant Game," IEEE Transactions on Signal Processing, 61.3:571-584, 2013. Article (CrossRef Link)

[16] Yu, D., Ning, L., Zou, Y., Yu, J., Cheng, X., \& Lau, F. C. M., "Distributed spanner construction with physical interference: constant stretch and linear sparseness," IEEE/ACM Transactions on Networking, PP(99), 1-14, 2017. Article (CrossRef Link)

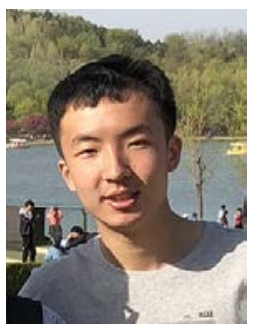

Junliang Chen received his B.E. degree in Electronic information science and technology from Jilin University. He is currently pursuing a master degree at Beihang University. His current research interests include cooperative cache and game theory.

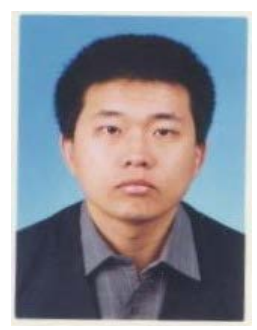

Gang Wang is currently an associate professor at Beihang University, Beijing, P. R. China. He received his $\mathrm{PhD}$ degree from Beihang University. His current research interests include wireless networks, with a particular focus on information dissemination and resource allocation.

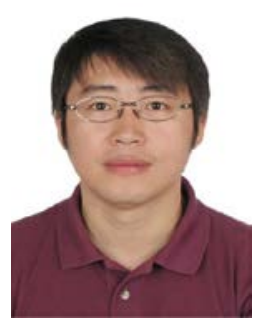

Fuxiang Wang is currently a lecturer at Beihang University, Beijing, P.R.China. He received his $\mathrm{PhD}$ degree from Beihang University. His current research interests include Blind signal processing and application of air traffic flow management. 